\title{
Uma Análise dos Ambientes de Ensino de Banco de Dados
}

\author{
Juliana Alves Pereira, Antônio Maria Pereira de Resende \\ Departamento de Ciência da Computação - Universidade Federal de Lavras (UFLA) \\ Caixa Postal 3037 - Lavras - MG - Brasil \\ julianaesistemas.ufla.br, tonioddcc.ufla.br
}

\begin{abstract}
This paper examined the Database educational software state of art, covering the techniques of Relational Algebra, Entity-Relationship Modeling, and SQL commands in order to understand the advantages and disadvantages offered by them. After a careful comparative analysis, the authors propose a model of teaching and learning environmental capturing the benefits of environments analyzed and correcting some of its disadvantages. The applicability of this work is based on the use of this new model in the teaching and learning process of Database discipline.
\end{abstract}

Resumo. Este artigo analisou o estado da arte de software educacionais de Banco de Dados, abrangendo as técnicas de Álgebra Relacional, Modelagem de Entidades e Relacionamentos, e Comandos $S Q L$, visando compreender as vantagens e desvantagens que elas propiciam. A partir dessa análise comparativa criteriosa, propôs-se um modelo de ambiente capturando as vantagens dos ambientes analisados e corrigindo algumas de suas desvantagens. A aplicabilidade deste trabalho apoia-se na utilização deste novo modelo no processo de ensino e aprendizagem de Banco de Dados.

\section{Introdução}

A sociedade atual passa por grandes mudanças, exigindo cidadãos críticos, criativos, reflexivos, com capacidade de aprender a aprender, de trabalhar em grupo, de se conhecer como indivíduo e como membro participante de uma sociedade que busca o seu próprio desenvolvimento, bem como o de sua comunidade. Cabe à educação formar este profissional [Nunes 2009].

O ritmo acelerado de inovações tecnológicas exige um sistema educativo capaz de estimular nos estudantes o interesse pela aprendizagem. Por esta razão, à educação não pode mais restringir-se ao conjunto de instruções que o professor transmite a um aluno passivo, mas deve enfatizar a construção do conhecimento pelo aluno e o desenvolvimento de novas competências necessárias para sobreviver na sociedade atual [Sancho 1998] [Nunes 2009].

Além disso, esse interesse diante de novos conhecimentos e técnicas deve ser mantido ao longo da sua vida profissional, que provavelmente, tenderá a se realizar em áreas diversas de um mercado cada vez mais sujeito ao impacto de novas tecnologias [Sancho 1998].

Com o intuito de produzir melhores resultados de aprendizagem e facilitar a compreensão de processos abstratos, faz-se necessária a atualização de metodologias de ensino diferenciadas. De acordo com Buzin em [Buzin 2001], o aluno mostra mais 
interesse por estas metodologias, pois, além de tornar o conteúdo mais atrativo, tais soluções minimizam dificuldades, melhoram o rendimento dos alunos, reproduzindo modelos da realidade de forma mais amigável em ambientes gráficos e interfaces ricas.

Nesse contexto, encontra-se um grande desafio para o ensino de futuros profissionais de qualidade para o exigente mercado atual. Portanto, o objetivo deste artigo é estudar a influência dos ambientes de ensino de Banco de Dados (BD), seus pontos positivos e negativos, comparando-os e propondo melhorias, através de uma melhor integração de seus recursos, a fim de se construir um modelo adequado de ambiente que apoie o aprendizado de BD.

Como objetivos específicos podem ser citados a) realizar um levantamento bibliográfico sobre os ambientes de ensino de $\mathrm{BD}$; b) analisar os pontos positivos e negativos destes ambientes; e c) propor um modelo de ambiente para o apoio ao aprendizado de BD.

As próximas seções deste trabalho dividem-se em: (i) Metodologia, onde se descreve os tipos de pesquisa e a forma de desenvolvimento do estudo; (ii) Desenvolvimento, que descreve todos os passos realizados para obtenção dos objetivos, apresentando um modelo de ambiente capaz de estimular e aumentar o aprendizado de BD; e, (iii) Conclusão, onde aborda os conhecimentos adquiridos e trabalhos futuros.

\section{Metodologia}

Para a consecução dos objetivos, o trabalho foi dividido nas etapas de: a) análise sistêmica dos ambientes de ensino de $\mathrm{BD}$; b) análise das vantagens e desvantagens dos ambientes de ensino de $\mathrm{BD}$; c) construção de uma proposta de modelo de ambiente de apoio ao ensino de BD.

Tabela 1 Etapas e sub-etapas da metodologia

\begin{tabular}{|l|l|}
\hline \multicolumn{1}{|c|}{ Etapas } & \multicolumn{1}{c|}{ Sub-etapas } \\
\hline $\begin{array}{l}\text { Análise Sistêmica dos } \\
\text { Ambientes de Ensino de Banco } \\
\text { de Dados }\end{array}$ & $\begin{array}{l}\text { a) aprender sobre as técnicas de Revisão Sistemática; b) aplicar a } \\
\text { revisão sistemática identificando artigos na área de ambientes virtuais } \\
\text { de ensino de BD; c) ler os artigos; e d) descrever os ambientes de } \\
\text { ensino identificados. }\end{array}$ \\
\hline $\begin{array}{l}\text { Análise das Vantagens e } \\
\text { Desvantagens dos Ambientes } \\
\text { de Ensino de Banco de Dados }\end{array}$ & $\begin{array}{l}\text { a) categorização de grupos de ambientes; e b) classificação dos } \\
\text { ambientes analisados quanto à sua aplicabilidade; e c) análise das } \\
\text { vantagens e desvantagens dos ambientes educacionais. }\end{array}$ \\
\hline $\begin{array}{l}\text { Construção de uma Proposta de } \\
\text { Modelo de Ambiente de Apoio } \\
\text { ao Ensino de Banco de Dados. }\end{array}$ & $\begin{array}{l}\text { a) levantamento de requisitos do novo modelo de ambiente de ensino; } \\
\text { b) elaboração do diagrama de blocos do novo modelo; e c) descrição } \\
\text { de cada módulo do diagrama de blocos. }\end{array}$ \\
\hline
\end{tabular}

\section{Desenvolvimento}

A aplicação da primeira até a última etapa da metodologia descrita acima levou seis meses a ser concluída, sendo que a atividade de levantamento de requisitos do novo modelo de ferramenta foi a que mais trabalho consumiu.

O projeto envolveu em média três pessoas e foi dividido em: análise dos ambientes de Álgebra Relacional (AR); análise dos ambientes Entidades 
Relacionamentos (ER) e linguagem SQL; e análise de técnicas de sintetizadores e reconhecedores de voz.

Após estas analises, realizou-se um levantamento de requisitos para um novo modelo de ambiente educacional de ensino para a disciplina de BD.

\subsection{Análise Sistêmica dos Ambientes de Ensino de Banco de Dados}

Durante esta etapa foi realizado um curso de Revisão Sistemática à distância e gratuito oferecido pela Unifesp [UNIFESP 2011]. Este curso foi de grande ajuda para reconhecimento das técnicas e compreensão dos artigos analisados.

O curso propiciou a identificação de artigos/trabalhos nesta área (Tabela 2), os quais foram de grande utilidade para compreensão do funcionamento de tais ambientes de ensino. Os artigos foram classificados de acordo com sua relevância (notas de 5 a 10) e então tomados para leitura.

Tabela 2 Classificação dos artigos/trabalhos encontrados

\begin{tabular}{|c|c|c|}
\hline Título & Apresentação & Relevância \\
\hline $\begin{array}{l}\text { An Intelligent SQL Tutor on the } \\
\text { Web }\end{array}$ & $\begin{array}{l}\text { International Journal of Artificial Intelligence in } \\
\text { Education (2003). Volume: 13, Issue: } 2-4 \text {, Publisher: } \\
\text { IOS Press Amsterdam, Pages: } 171-195 \text {. }\end{array}$ & 10 \\
\hline $\begin{array}{l}\text { SQLator - An Online SQL } \\
\text { Learning Workbench }\end{array}$ & $\begin{array}{l}\text { ACM SIGCSE Bulletin (2004). New York, NY, USA. } \\
\text { Volume: 36, Issue: 3, Pages: 223-227. }\end{array}$ & 10 \\
\hline $\begin{array}{l}\text { Advanced Database Concepts for } \\
\text { Undergraduates: Experience with } \\
\text { Teaching a Second Course }\end{array}$ & $\begin{array}{l}\text { SIGCSE ACM Special Interest Group on Computer } \\
\text { Science Education (2001), New York, NY, USA, Pages: } \\
357-361 \text {. }\end{array}$ & 9 \\
\hline $\begin{array}{l}\text { Automated tutoring for a database } \\
\text { skills training environment. }\end{array}$ & $\begin{array}{l}\text { SIGCSE } 2005 \text { - 36th SIGCSE Technical symposium on } \\
\text { Computer Science Education, 23-27 February 2005, St. } \\
\text { Louis, USA. } 5 \text { p. }\end{array}$ & 10 \\
\hline $\begin{array}{l}\text { Acharya: An Intelligent Tutoring } \\
\text { Environment for Learning SQL }\end{array}$ & $\begin{array}{l}\text { In Proceedings of the Vidyakash (2002), International } \\
\text { Conference on Online Learning, Mumbai, India. }\end{array}$ & 10 \\
\hline 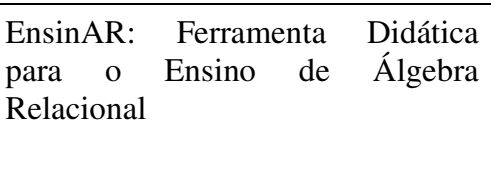 & $\begin{array}{l}\text { Programa de Pós-Graduação. Universidade Federal de } \\
\text { Santa Catarina. Departamento de Informática e } \\
\text { Estatística. Curso de Ciências da Computação. } 151 \\
\text { págs. }\end{array}$ & 10 \\
\hline $\begin{array}{lrr}\text { Using Games-Based } & \text { eLearning } \\
\text { Technologies in } & \text { Overcoming } \\
\text { Difficulties in } & \text { Teaching } \\
\text { Information Systems } & \end{array}$ & $\begin{array}{l}\text { Journal of Information Technology Education, v5 p459- } \\
4762006 .\end{array}$ & 8 \\
\hline $\begin{array}{l}\text { Using an Online Games-Based } \\
\text { Learning Approach to Teach } \\
\text { Database Design Concepts }\end{array}$ & $\begin{array}{l}\text { The Electronic Journal of e-Learning, Volume } 4 \text { Issue } 1 \text {, } \\
\text { 2006. pp 103-110. }\end{array}$ & 10 \\
\hline \begin{tabular}{lcr}
\multicolumn{3}{c}{ iDFQL - Uma Ferramenta de Apoio } \\
ao Processo & de & Ensino- \\
Aprendizagem & da & Álgebra \\
Relacional & Baseado & no \\
Construcionismo & &
\end{tabular} & $\begin{array}{l}\text { I Workshop de Educação em Informática RJ/ES - I WEI } \\
\text { RJ/ES (2004). Vitória, ES, Brasil. }\end{array}$ & 10 \\
\hline $\begin{array}{l}\text { LEaRning: Uma } \quad \text { Ferramenta } \\
\text { Didática para Projeto Conceitual de } \\
\text { Banco de Dados }\end{array}$ & $\begin{array}{l}\text { [SMSI] - Anais do Simpósio Mineiro de Sistemas de } \\
\text { Informação II: } 2005 \text { Out. } 20 \text { - 21: Belo Horizonte - } \\
\text { MG. }\end{array}$ & 10 \\
\hline $\begin{array}{l}\text { Mining Relational Databases with } \\
\text { Multi-view Learning }\end{array}$ & $\begin{array}{l}\text { Proceeding MRDM '05 Proceedings of the 4th } \\
\text { international workshop on Multi-relational mining. }\end{array}$ & 6 \\
\hline
\end{tabular}




\begin{tabular}{|c|c|c|}
\hline & ACM New York, NY, USA (2005). Pages 15-54. & \\
\hline Intérprete de Álgebra Relacional & $\begin{array}{l}\text { International Journal of Computer Theory and } \\
\text { Engineering, Vol.3, No.1, February, 2011. 1793-8201. }\end{array}$ & 10 \\
\hline $\begin{array}{l}\text { WinRDBI: A Windows-based } \\
\text { Relational Database Educational } \\
\text { Tool }\end{array}$ & $\begin{array}{l}\text { Proceedings of the 28th ACM SIGCSE Technical } \\
\text { Symposium on Computer Science Education. San Jose, } \\
\text { California, February } 27 \text { - March 1, 1997, pp. 126-130. }\end{array}$ & 10 \\
\hline $\begin{array}{l}\text { SimAlg: Implementação de um } \\
\text { simulador de consultas em Álgebra } \\
\text { Relacional }\end{array}$ & $\begin{array}{l}\text { Trabalho de Graduação apresentado ao Curso de } \\
\text { Ciência da Computação da Universidade Federal de } \\
\text { Santa Maria (UFSM, RS), como requisito parcial para a } \\
\text { obtenção do grau de Bacharel em Ciência da } \\
\text { Computação. }\end{array}$ & 10 \\
\hline $\begin{array}{l}\text { A aprendizagem através da prática } \\
\text { do modelo para o ensino de bancos } \\
\text { de dados avançados }\end{array}$ & $\begin{array}{l}\text { Proceedings da conferência Australasian sobre } \\
\text { Computing educação. ACM New York, NY, EUA } \\
\text { (2000). }\end{array}$ & 9 \\
\hline $\begin{array}{l}\text { SQL OnLine, ambiente de apoio ao } \\
\text { ensino de Banco de Dados }\end{array}$ & $\begin{array}{l}\text { Copyright 1998. Associação para o Avanço da } \\
\text { Informática na Educação (AACE). Distribuição através } \\
\text { da Web com a permissão da AACE. }\end{array}$ & 10 \\
\hline $\begin{array}{l}\text { Supporting Active Database } \\
\text { Learning and Training through } \\
\text { Interactive Multimedia }\end{array}$ & $\begin{array}{l}\text { Ciência de Instrução (2005). Volume: 33, Issue: 1, } \\
\text { Publisher: Kluwer Academic Publishers, Pages: } 73-95 \\
\text { Volume: 33, Issue: } 1 \text { Editora: Kluwer Academic } \\
\text { Publishers, Páginas: } 73-95 \text {. }\end{array}$ & 10 \\
\hline $\begin{array}{l}\text { SPARSE: Um Ambiente de Ensino } \\
\text { e Aprendizado de Engenharia de } \\
\text { Software Baseado em Jogos e } \\
\text { Simulação }\end{array}$ & $\begin{array}{l}\text { Brazilian Symposium on Computer in Education } \\
\text { (SBIE). João Pessoa - PB, } 23 \text { a } 26 \text { de Novembro de } \\
2010 \text {. }\end{array}$ & 8 \\
\hline $\begin{array}{l}\text { Ferramenta para Correção } \\
\text { Automática de Exercícios para a } \\
\text { Linguagem SQL - QuicklySQL }\end{array}$ & $\begin{array}{l}\text { Relatório apresentado à banca examinadora do Trabalho } \\
\text { de Conclusão do Curso de Ciência da Computação para } \\
\text { análise e aprovação. Universidade do Vale do Itajaí. } \\
\text { Centro de Ciências Tecnológicas da Terra e do Mar. }\end{array}$ & 10 \\
\hline $\begin{array}{l}\text { KERMIT: A Knowledge-based } \\
\text { Entity Relationship Modelling } \\
\text { Intelligent Tutor }\end{array}$ & $\begin{array}{l}\text { Conferência Zelândia Computer Estudantes Pesquisa } \\
\text { em Ciências (NZCSRSC), Christchurch, Nova } \\
\text { Zelândia, 2001. }\end{array}$ & 10 \\
\hline $\begin{array}{l}\text { AprenDER: Ferramenta de Apoio à } \\
\text { Construção de Diagrama Entidade } \\
\text { Relacionamento para Deficientes } \\
\text { Visuais }\end{array}$ & $\begin{array}{l}\text { SBIE 2010. Sociedad Brasileña de Computación (SBC) } \\
\text { de } 23 \text { a } 26 \text { de noviembre de } 2010 \text {. Universidade Federal } \\
\text { de Paraíba, Universidade Federal de Pernambuco e } \\
\text { Empresa Municipal de Informática - EMPREL Recife. }\end{array}$ & 10 \\
\hline $\begin{array}{lll}\text { Aprendizagem em Banco } & \text { de } \\
\text { Dados: Implementação } & \text { de } \\
\text { Ferramenta de Modelagem E.R. }\end{array}$ & $\begin{array}{l}\text { Monografia submetida à Universidade Federal de Santa } \\
\text { Catarina em convenio com a Universidade de Várzea } \\
\text { Grande como parte dos requisitos para a obtenção do } \\
\text { grau de especialista em BD. }\end{array}$ & 10 \\
\hline
\end{tabular}

\subsection{Análise das Vantagens e Desvantagens dos Ambientes de Ensino de Banco de Dados}

Foram analisados 17 ambientes de ensino de BD, entretanto, a descrição dos ambientes de ensino possibilitou a categorização em grupos e posteriormente sua classificação.

A classificação destas ferramentas foi realizada quanto a sua aplicabilidade, ou seja, dentre todos os ambientes analisados e descritos, muitos deles apresentaram grandes semelhanças e algumas de suas funcionalidades já haviam sido abordadas por outros ambientes, contudo somente os ambientes considerados como mais completos (contidos na Tabela 3) foram objetivos de análise. 
Tabela 3 Ferramentas analisadas

\begin{tabular}{|c|l|}
\hline \multicolumn{1}{|c|}{ Grupo } & \multicolumn{1}{|c|}{ Ferramentas Educacionais } \\
\hline \multirow{2}{*}{$\begin{array}{c}\text { Entidade Relacionamento } \\
(\text { ER })\end{array}$} & AprenDER \\
\cline { 2 - 2 } & Kermit \\
\cline { 2 - 2 } $\begin{array}{c}\text { Álgebra Relacional } \\
(\text { AR) }\end{array}$ & BrModelo \\
\cline { 2 - 2 } & EnsiNAR \\
\cline { 2 - 2 } & Intérprete de Álgebra Relacional \\
\cline { 2 - 2 } & SimAlg \\
\hline \multirow{2}{*}{$\begin{array}{c}\text { Linguagem SQL } \\
\end{array}$} & SQL OnLine \\
\cline { 2 - 2 } & SQLator \\
\cline { 2 - 2 } & SQL-Tutor \\
\hline
\end{tabular}

\subsubsection{Ambientes de Ensino Entidade Relacionamento}

Independentemente da área, percebe-se a necessidade de armazenar e manipular dados e neste contexto é fundamental que estes sejam armazenados de forma organizada e permitam um posterior acesso eficiente. Neste sentido, torna-se necessário projetar um BD [Magalhães e Neto 2007].

Para a realização do projeto de um BD podem ser utilizados vários modelos de dados como ferramentas de projeto. Dentre os modelos de dados conceituais, o mais conhecido é o modelo ER, proposto por Chen [Chen 1976]. Esse modelo caracteriza-se por descrever a estrutura do BD como uma coleção de entidades (objetos existentes no universo de discurso) e relacionamentos (associações entre duas ou mais entidades), utilizando uma notação gráfica [Ferreira e Arantes 2005].

O modelo ER permite a descrição do subconjunto do mundo real que será retratado no $\mathrm{BD}$ com um alto nível de abstração, sem a preocupação com detalhes de armazenamento [Magalhães e Neto 2007]. Oferecendo assim, suporte em adquirir conhecimentos e habilidades para realizar projetos de desenvolvimento de BD.

Os ambientes selecionados para análise foram: AprenDER, KERMIT e BrModelo. A Tabela 4 contém as vantagens e desvantagens destes ambientes.

Tabela 4 Características dos ambientes ER

\begin{tabular}{|c|c|c|c|}
\hline Ambiente & Categoria & Vantagens & Desvantagens \\
\hline AprenDER & ER & $\begin{array}{l}\text { Gravação do problema a ser modelado } \\
\text { (sintetizador de voz); } \\
\text { À medida que o usuário navega entre as } \\
\text { opções é disparada uma mensagem de } \\
\text { voz, informando qual o item } \\
\text { selecionado; } \\
\text { Os comandos e mensagens falados pelo } \\
\text { sistema são reproduzidos na língua } \\
\text { portuguesa. }\end{array}$ & $\begin{array}{l}\text { Não cobre todos os elementos } \\
\text { gráficos possíveis para a elaboração } \\
\text { completa de um diagrama; } \\
\text { Limita o número máximo de } \\
\text { entidades possíveis para } 9 \text { (nove); } \\
\text { Limita para } 5 \text { o número de atributos } \\
\text { por entidade; } \\
\text { Ambiente restrito as descrições } \\
\text { predeterminadas; } \\
\text { Não possui uma visualização dos } \\
\text { comandos SQL. }\end{array}$ \\
\hline Kermit & ER & $\begin{array}{l}\text { É dado ao aluno um estudo de caso e ele } \\
\text { cria sua modelagem ER para o problema } \\
\text { proposto, que atenda aos requisitos } \\
\text { especificados; } \\
\text { O sistema seleciona um novo problema }\end{array}$ & $\begin{array}{l}\text { O aluno não tem a oportunidade de } \\
\text { se referir ao texto do feedback, ou } \\
\text { seja, se interagir com o agente } \\
\text { animado; } \\
\text { Não possui uma visualização dos }\end{array}$ \\
\hline
\end{tabular}




\begin{tabular}{|c|c|c|c|}
\hline & & $\begin{array}{l}\text { que melhor se adeque ao modelo do } \\
\text { aluno; } \\
\text { São disponíveis seis níveis de feedback; } \\
\text { O sistema de ensino incorpora um } \\
\text { agente animado (o gênio). }\end{array}$ & $\begin{array}{l}\text { comandos SQL; } \\
\text { Não possui tutoriais. }\end{array}$ \\
\hline BrModelo & ER & $\begin{array}{l}\text { Apresenta uma interface muito simples e } \\
\text { funcional; } \\
\text { Permite alterações estruturais no modelo } \\
\text { diante de novas decisões do analista; } \\
\text { Contem dicionário de dados completos, } \\
\text { específico para cada objeto do esquema } \\
\text { e com capacidade de reunião em um } \\
\text { único documento; } \\
\text { Permite a interação com o usuário no } \\
\text { momento da conversão (conceitual para } \\
\text { lógico). }\end{array}$ & $\begin{array}{l}\text { Não é dada uma descrição textual } \\
\text { e/ou com áudio dos requisitos ao } \\
\text { aluno; } \\
\text { Não possui uma sessão do aluno. }\end{array}$ \\
\hline
\end{tabular}

\subsubsection{Ambientes de Ensino de Álgebra Relacional}

Este ambiente é característico por seu processo de construção da consulta, o que proporciona um maior envolvimento do aluno com os conceitos algébricos a serem aprendidos [Appel e Traina Jr. 2004].

Por ser uma linguagem de consulta visual, o ambiente permite aos alunos aumentar seu entendimento sobre o relacionamento entre a AR e a linguagem SQL, tornando o processo de ensino-aprendizagem mais interativo e estimulante.

Os ambientes selecionados para análise foram: EnsinAR, Intérprete de Álgebra Relacional e SimAlg. A Tabela 5 contém as vantagens e desvantagens destes ambientes.

Tabela 5 Características dos ambientes de Álgebra Relacional

\begin{tabular}{|c|c|c|c|}
\hline Ambiente & Categoria & Vantagens & Desvantagens \\
\hline EnsinAR & AR & $\begin{array}{l}\text { Para cada operação de AR associou-se } \\
\text { um botão, que ao ser pressionado } \\
\text { escreve no prompt de execução o } \\
\text { símbolo correspondente à operação; } \\
\text { É possível executar todas as operações } \\
\text { fundamentais de AR; } \\
\text { Realiza análises léxicas, sintáticas e } \\
\text { semânticas na expressão de entrada; } \\
\text { É possível realizar operações com } \\
\text { tabelas próprias; } \\
\text { Visualização da sintaxe SQL. }\end{array}$ & $\begin{array}{l}\text { Para escrever os comandos em AR, que } \\
\text { usam letras gregas, adaptou-se uma } \\
\text { fonte já existente do Windows. Não } \\
\text { utilizando a mesma notação ensinada } \\
\text { nos livros e por professores; } \\
\text { Não é dada uma descrição textual e/ou } \\
\text { com áudio dos requisitos ao aluno } \\
\text { (sessão do aluno); } \\
\text { Não possibilita visualização do retorno } \\
\text { dos dados da consulta; } \\
\text { Não possui tutoriais. }\end{array}$ \\
\hline $\begin{array}{l}\text { Intérprete } \\
\text { de } \\
\text { Álgebra } \\
\text { Relacional }\end{array}$ & $\mathrm{AR}$ & $\begin{array}{l}\text { Possui uma interface simples e fácil de } \\
\text { manipular; } \\
\text { Feedbacks imediatos são dados aos } \\
\text { usuários sobre as consultas realizadas; } \\
\text { Além da consulta em AR é possível } \\
\text { visualizá-la convertida em SQL. }\end{array}$ & $\begin{array}{l}\text { Não suporta todos os operandos de AR; } \\
\text { Usa-se BD Oracle, não possuindo } \\
\text { portabilidade para outros BD; } \\
\text { Não é dada uma descrição textual e/ou } \\
\text { com áudio dos requisitos. }\end{array}$ \\
\hline SimAlg & $\mathrm{AR}$ & $\begin{array}{l}\text { A ferramenta utiliza de um BD que não } \\
\text { requer instalação nem configuração; } \\
\text { Realiza uma avaliação léxica e }\end{array}$ & $\begin{array}{l}\text { Dirigida para a plataforma Windows; } \\
\text { As tabelas e os dados já estão } \\
\text { armazenados. A ferramenta não permite }\end{array}$ \\
\hline
\end{tabular}




\begin{tabular}{|l|l|l|}
\hline & $\begin{array}{l}\text { sintática; } \\
\text { Disponibiliza feedback de erros e um } \\
\text { menu de ajuda; } \\
\text { Exibe passo a passo a construção das } \\
\text { queries. }\end{array}$ & $\begin{array}{l}\text { a flexibilidade do usuário nas consultas; } \\
\text { É necessário que o usuário realize varias } \\
\text { consultas simples para que chegue a } \\
\text { uma consulta complexa; } \\
\text { Suporta apenas as operações: seleção, } \\
\text { projeção, união, intersecção, diferença, } \\
\text { produto cartesiano e junção natural. }\end{array}$ \\
\hline
\end{tabular}

\subsubsection{Ambiente de Ensino da Linguagem SQL}

Faz parte do conteúdo programático da disciplina de BD nos vários cursos de graduação em computação, uma abordagem consistente de SQL (Structured Query Language) [Souza 2004]. SQL é a linguagem mais aceita e amplamente utilizada para definir, manipular e controlar SGBDs relacionais, com a capacidade de formular perguntas muito complexas, sua natureza declarativa a torna bastante difícil para muitos alunos entender [Sadiq, Orlowska e outros 2004].

Faz-se necessário a criação de ambientes que possibilitem a criação/remoção de elementos estruturais; realizem a alimentação da Base de Dados; e implementem operações de manipulação.

Os ambientes selecionados para análise foram: SQL OnLine, SQLator e SQLTutor. A Tabela 6 contém as vantagens e desvantagens destes ambientes.

Tabela 6 Características dos ambientes de linguagem SQL

\begin{tabular}{|c|c|c|c|}
\hline Ambiente & Categoria & Vantagens & Desvantagens \\
\hline $\begin{array}{c}\text { SQL } \\
\text { OnLine }\end{array}$ & SQL & $\begin{array}{l}\text { Mantém um BD com exercícios classificados } \\
\text { pelo grau de complexidade; } \\
\text { As perguntas são sorteadas dentre as questões } \\
\text { ainda não respondidas; } \\
\text { Mostra-se um modelo físico de dados para } \\
\text { ilustrar o contexto da pergunta; } \\
\text { Possibilita classificação geral do aluno em } \\
\text { relação aos colegas e uma análise histórica e } \\
\text { estatística das questões respondidas. }\end{array}$ & $\begin{array}{l}\text { O aluno não vê o resultado da } \\
\text { consulta; } \\
\text { O ambiente não possui as opções } \\
\text { de construção e remoção de } \\
\text { tabelas, atributos e relações, } \\
\text { alimentação do BD, entre outros; } \\
\text { Ambiente restrito a descrições } \\
\text { predeterminadas; } \\
\text { O ambiente não possui um } \\
\text { manual de ajuda. }\end{array}$ \\
\hline SQLator & SQL & $\begin{array}{l}\text { Possui painéis, videoconferência e salas de } \\
\text { chat; } \\
\text { Descrições das bases de dados; } \\
\text { Classificação das consultas por categorias; } \\
\text { Capacidade de escrever e executar qualquer } \\
\text { consulta SQL em determinada Base de } \\
\text { Dados; } \\
\text { Monitoramento do progresso individual e do } \\
\text { grupo, através de relatórios de status e por } \\
\text { meio de estatísticas. }\end{array}$ & $\begin{array}{l}\text { O ambiente não possui as opções } \\
\text { de construção e remoção de } \\
\text { tabelas, atributos e relações, bem } \\
\text { como a alimentação do BD; } \\
\text { O ambiente não possui um } \\
\text { manual de ajuda; }\end{array}$ \\
\hline $\begin{array}{l}\text { SQL- } \\
\text { Tutor }\end{array}$ & SQL & $\begin{array}{l}\text { Fácil de usar; } \\
\text { Para cada aluno é atribuído um nível, na } \\
\text { primeira interação do aluno com o sistema e } \\
\text { este nível é posteriormente atualizado; } \\
\text { Adapta-se as ações dos usuários e ao seu } \\
\text { conhecimento e habilidades de aprendizagem; }\end{array}$ & $\begin{array}{l}\text { O ambiente não possui as opções } \\
\text { de construção e remoção de } \\
\text { tabelas, atributos e relações, } \\
\text { alimentação do BD, entre outros; } \\
\text { Ambiente restrito a descrições } \\
\text { predeterminadas. }\end{array}$ \\
\hline
\end{tabular}




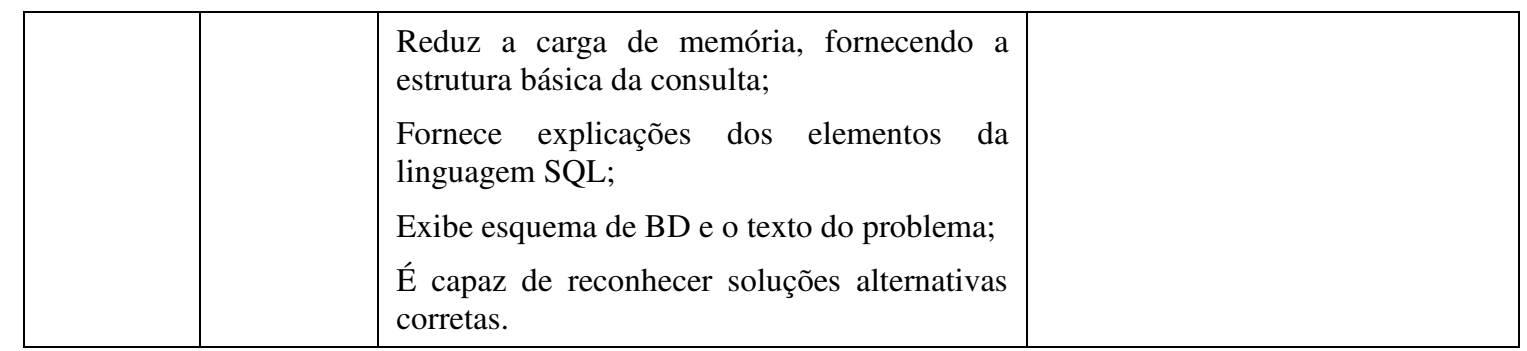

\subsection{Construção de uma Proposta de Modelo de Ambiente de Apoio ao Ensino de Banco de Dados.}

As tabelas Tabela 4, Tabela 5 e Tabela 6 nos mostram que os ambientes não contemplam de todas as atividades necessárias para desenvolvimento de um BD. A maioria dos ambientes analisados trata-se de exercícios com a linguagem SQL, outra parte corresponde a exercícios do tipo ER e AR, sendo uma minoria a integração de diversas técnicas de $\mathrm{BD}$ acoplados em um único ambiente.

A fim de proporcionar ao aluno um sequenciamento das atividades realizadas em um único ambiente, é proposto o desenvolvimento de um ambiente web interativo que atenda todo o ciclo de vida de um $\mathrm{BD}$, desde a análise de requisitos à implementação, combinando o conhecimento de aprendizagem e treinamento de habilidades em um ambiente integrado. A integração destes componentes é fundamental para que o aluno possa aprender, treinar e desenvolver seus conhecimentos dentro do mesmo ambiente [Pahl, Barret e Kenny 2004].

A Figura 1apresenta o diagrama de blocos do novo modelo e a descrição de cada um de seus módulos. Os módulos propostos são: exercícios com questões teóricas, criação do modelo ER, criação do modelo lógico, criação do esquema físico de dados, utilização da linguagem SQL e uso das técnicas de AR. Em todos os módulos descritos há: tutoriais multimídias, interação de voz do agente animado com o usuário, acesso a um painel de dúvidas, conversação através de chat entre os usuários do ambiente, visualização de feedback/sugestões dos exercícios e visualização de análises históricas.

Para que o aluno compreenda as teorias, os modelos e conceitos (análise de requisitos, modelo ER, normalização, $\mathrm{AR}$, modelo lógico, linguagem $\mathrm{SQL}$, entre outros) serão disponibilizados: painéis; vídeo conferência; sala de chats e tutoriais multimídia (vídeos e animações). A junção de técnicas de comunicação síncrona e assíncrona tem como objetivo um maior suporte para a aprendizagem, estimulando e criando oportunidades para que o estudante pense em ideias sobre várias perspectivas.

Como existem várias maneiras corretas de se criar um diagrama ER, é necessário sua conversão para o esquema lógico para uma correta validação do modelo. O modelo físico tem como objetivo introduzir ao usuário durante a realização de exercícios SQL e exercícios de AR o projeto do $\mathrm{BD}$, para que o mesmo possa manipulálo adequadamente.

Com base em toda a modelagem do BD feita durante a resolução dos exercícios dos módulos anteriores o módulo SQL permite a criação/remoção de elementos estruturais, alimentação da Base de Dados, implementação de operações de atualizações e consultas, e o módulo AR disponibiliza a criação de consultas, através de ícones do modelo e seus relacionamentos. 
As técnicas de engenharia reversa têm como papel principal possibilitar a alteração, manipulação e remoção de algumas bases de dados já criadas e alimentadas no sistema. Hoje, são diversas as situações em que se veem necessárias manutenções de base de dados já consolidadas e em utilização em uma organização.

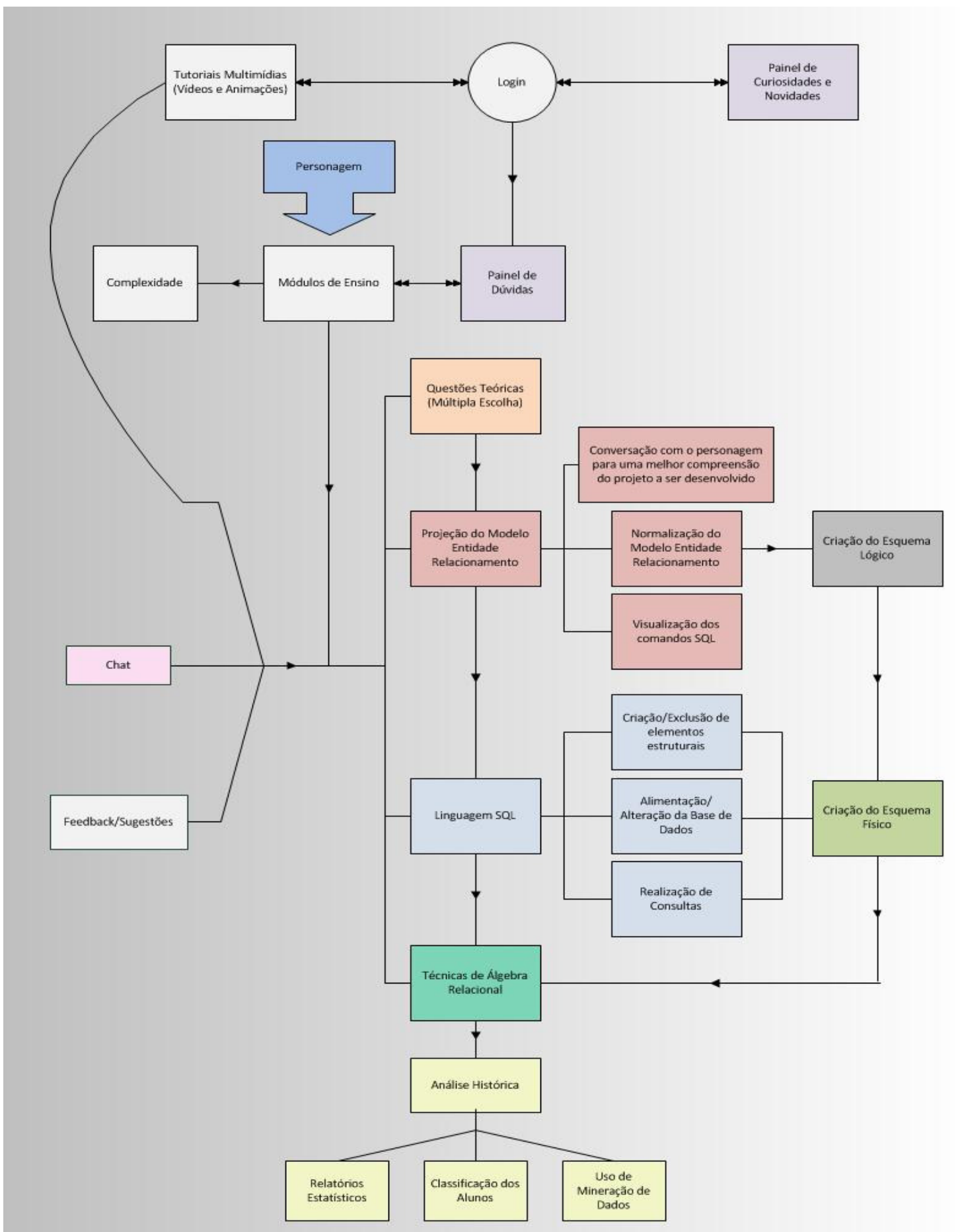

Figura 1 - Proposta de Modelo de Ambiente de Apoio ao Ensino de BD

O feedback apresentado por todos os módulos listados, serão compostos por erro de sinalização (sistema exibe automaticamente) e sugestões (indicar a parte da consulta 
que está causando um erro), a fim de permitir aos usuários refletirem sobre os resultados obtidos, depurar suas ideias por intermédio da busca de novos conteúdos e novas estratégias.

O material será dividido em módulos de conhecimento, estruturado hierarquicamente em diferentes níveis. Cada módulo possui diversos conceitos e cada conceito é composto de um ou mais requisitos. Os exercícios serão classificados de acordo com o grau (auto, médio ou baixo) de complexidade e associado a cada exercício será apresentado uma hierarquia de sugestões, com orientações de como se aproximar da solução correta, enriquecendo a experiência de aprendizagem e explorando diferenças individuais de conhecimento e habilidades de cada um de seus usuários.

Um motor inteligente que avalia as consultas SQL dos alunos (algoritmos heurísticos) e um modelador do aluno (Modelo Baseado em Conhecimento) serão implementados. O ambiente registrará todas as respostas enviadas pelos alunos, possibilitando uma análise histórica e estatística das questões, e uma classificação dos alunos. A classificação dos alunos tenta desafiar e motivar o aprendiz, envolvendo-o em uma competição saudável.

Além das perguntas pré-definidas disponíveis pelo professor no ambiente, o usuário poderá criar seu próprio projeto de BD ou carregar uma Base de Dados para realização de consultas.

Contudo, a fim de possibilitar o aprendizado pela prática, um cenário autêntico, similar ao cenário encontrado no mercado de trabalho, será disponibilizado pelo ambiente de ensino modelado, fornecendo medidas significativas de avaliação do aluno no decorrer da disciplina.

\section{Conclusões}

A disciplina de BD é uma disciplina obrigatória na grade curricular da maioria dos cursos de computação no Brasil. Essa disciplina normalmente aborda assuntos que compreendem conteúdos essenciais para o desenvolvimento do aluno como profissional da área [Appel e Traina Jr. 2004].

Muitos dos assuntos são abordados por meio da tutoria humana individual, método eficaz de ensino, mas que não prove recursos suficientes para cada aluno [Suraweera e Mitrovic 2004]. Contudo, este artigo objetivou a modelagem de um sistema inteligente de ensino que ofereça boas perspectivas para o fornecimento de conhecimento individualizado, através de sessões pedagógicas, abrangendo uma população muito maior de alunos. Estes ambientes são vistos como um meio ideal de disseminação de conteúdo, onde os alunos podem atuar como colaboradores no processo de ensino-aprendizagem dos demais colegas [Appel e Traina Jr. 2004].

Para consecução dos objetivos, iniciaram-se as atividades pelo estudo e aplicação de uma revisão sistemática dos ambientes de apoio ao ensino de BD, realizando-se análise de 17 ambientes.

A partir das descrições dos ambientes, de suas vantagens e desvantagens, observou-se que um dos maiores benefícios dos ambientes analisados é o auxílio aos profissionais na apresentação didática, proporcionando o controle dos alunos fora da sala de aula, podendo desta maneira identificar e avaliar os pontos que precisam ser reforçados. 
Constatou-se que as ferramentas analisadas não oferecem suporte ao ensino de BD cobrindo todo o processo de desenvolvimento de $\mathrm{BD}$, o que dificulta o aprendizado do aluno, por não poder seguir uma linha de raciocínio. $O$ aluno é, constantemente, forçado a utilizar vários softwares para aprender sobre conceitos, modelagem e implementação completa de um BD específico.

Como resultado, propôs-se um novo modelo de ambiente de apoio ao ensino de $\mathrm{BD}$, contendo os módulos: exercícios com questões teóricas, criação do modelo ER (protótipo desenvolvido), criação do modelo lógico, criação do esquema físico de dados, utilização da linguagem SQL (protótipo desenvolvido) e uso das técnicas de AR; cujas funcionalidades foram detalhadas em um diagrama de blocos elaborado (Figura $1)$.

Dentre os trabalhos futuros, propõe-se o desenvolvimento de um software que contemple o modelo proposto. Propõe-se também, o uso de mineração de dados no ambiente desenvolvido dentre outras técnicas para suporte e acompanhamento da relação ensino/aprendizado, a fim de descobrir padrões de aprendizagem e de formação, pois de acordo com [Souza 2004] cada aluno tem os seus hábitos e preferências e o ambiente deve utilizar-se várias estratégias com intuito de atingir o maior número possível de alunos.

\section{Referências}

Appel, Ana Paula; Traina Jr., Caetano. iDFQL - Uma Ferramenta de Apoio ao Processo de Ensino-Aprendizagem da Álgebra Relacional Baseado no Construcionismo. Instituto de Ciências Matemáticas e de Computação - USP. São Carlos, SP. 200414 p.

Buzin, Paulo F.W. Keglevich. A epistemologia da Ciência da Computação: Desafio do Ensino dessa Ciência. In: Revista de Educação, Ciência \& Cultura - Centro Universitário La Salle, Vol. 6, n. 2, Canoas/RS, p.7-33, 2001.

Cândido, Carlos Henrrique. Aprendizagem em Banco de Dados: Implementação de Ferramenta de Modelagem E.R. Universidade Federal de Santa Catarina (UNIVAG) - Pós-Graduação em Banco De Dados. 2005. 44 p.

Ferreira, Janaína Liziane; Arantes, Álisson Rabelo. LEaRning: Uma Ferramenta Didática para Projeto Conceitual de Bancos de Dados. Pontifícia Universidade Católica de Minas Gerais - Brasil. 2005. 6 p.

Peter Pin-Shan Chen. 1976. The entity-relationship modell—toward a unified view of data. ACM Trans. Database Syst. 1, 1 (March 1976), 9-36. DOI=10.1145/320434.320440 http://doi.acm.org/10.1145/320434.320440 apud [Ferreira e Arantes 2005]

Lautert, Larissa R. Implementação de um Simulador de Consultas em Àlgebra Relacional. Trabalho de Graduação. Universidade Federal de Santa Maria. Centro de Tecnologia. Curso de Ciência da Computação. Santa Maria, RS, Brasil. 2010. 46 p.

Magalhães, Rafael L.; Neto, Michelle M. F. AprenDER: Ferramenta de Apoio à Construção de Diagrama Entidade Relacionamento para Deficientes Visuais. Coordenação de Informática. Instituto Federal Fluminense (IFF). 2007. 10 p. 
Mitrovic, Antonija. An Intelligent SQL Tutor on the Web. Intelligent Computer Tutoring Group. Computer Science Department University of Canterbury - New Zealand. International Journal of Artificial Intelligence in Education 13 (2003) 171195.

Nunes, Irene Albertina Hugen. Curso de Especialização em Mídias na Educação. Capacitação para docentes da E.E.B. Manoel Cruz - Mídias na Sala de Aula. Universidade Federal do Rio Grande. São Joaquim, SC. 39 p. 2009

Paes, Ederson Luiz. EnsinAR: Ferramenta Didática para o Ensino de Álgebra Relacional. Universidade Federal de Santa Catarina - Centro Tecnológico. Departamento de Informática e Estatística. Florianópolis. 2004. 14 p.

Pahl, Claus; Barrett, Ronan; Kenny, Claire. Supporting Active Database Learning and Training Through Interactive Multimedia. Annual Joint Conference Integrating Technology into Computer Science Education. Leeds, United Kingdom, p. 27-31, 2004.

Sadiq, Shazia; Orlowska Maria; Sadiq, Wasim; LIN Joe. SQLator - An Online SQL Learning Workbench. School of Information Technology and Electrical. Annual Joint Conference Integrating Technology into Computer Science Education. Leeds, United Kingdom, p. 223-227, 2004.

Sancho, J. M. Para uma Tecnologia Educacional. ArtMed, 1998.

Souza, André Luiz. SQL OnLine, Ambiente de Apoio ao Ensino de Banco de Dados. Faculdades Associadas de Uberaba - FAZU. 20049 p.

Suraweera, Pramuditha; Mitrovic, Antonija. An Intelligent Tutoring System for Entity Relationship Modelling. Intelligent Computer Tutoring Group Department of Computer Science, University of Canterbury - New Zealand. International Journal of Artificial Intelligence in Education. Volume 14 Issue 3,4, December 2004. 44 p.

Universidade Federal de São Paulo. Curso de Revisão Sistemática à Distância e Gratuito Oferecido pela UNIFESP. Disponível em http://www.virtual.epm.br/cursos/metanalise/. Acesso em: 26/04/2011. 